\title{
Serial perfusion brain tomographic scans detect reversible focal ischaemia in Rasmussen's encephalitis
}

\author{
Sobhan Vinjamuri, J P Leach, I K Hart
}

\begin{abstract}
Summary
A 39-year-old man with advanced adultonset Rasmussen's encephalitis was treated with prednisolone and long-term, high-dose, human intravenous immunoglobulin. A pretreatment, semiquantitative interictal brain perfusion single photon emission computed tomography (SPECT) scan using ${ }^{99} \mathrm{Tc}^{\mathrm{m}}$ HMPAO (hexamethylene propylene amine oxime) showed hypoperfusion in the clinically affected right frontal, parietal and temporal lobes and contralateral perfusion defects. A second scan 8 months later revealed significant improvements (more than two standard deviations) in perfusion of the right frontal and temporal lobes despite serial magnetic resonance imaging evidence of permanent brain damage. This was associated with useful recovery of the patient's physical and cognitive function. We conclude that serial perfusion brain SPECT scanning is a useful method to demonstrate improvement in patients with Rasmussen's encephalitis in response to therapy.
\end{abstract}

Keywords: brain; SPECT scanning; Rasmussen's encephalitis

Rasmussen's encephalitis (RE) is a rare, progressive autoimmune disease that causes focal, confluently spreading, destruction of the brain. First described by Rasmussen et al in 1958 , it is characterised by frequent and severe epileptic seizures, hemiparesis, and cognitive decline. ${ }^{1}$ Diagnosed on brain biopsy, it usually affects one cerebral hemisphere and is commonly seen in children under 10 years of age, although adults can also develop this disorder. Magnetic resonance imaging (MRI) shows changes of cerebral atrophy in the affected hemisphere.

Surgical resection is the conventional treatment. $^{2}{ }^{3}$ More recently, immunomodulatory therapies including plasmapheresis and human intravenous immunoglobulins (hIVIg), have been used in attempts to modify or halt the progression of the disease. ${ }^{4}$ Although these therapies are still experimental, pilot studies suggest that patients need to continue on hIVIg therapy for many years, and possibly for the rest of their lives. ${ }^{5}$ When evaluating such novel and expensive treatments, it is essential to have well-designed protocols with a variety of objec- tive outcome measures to monitor brain function serially. Clinical assessment is often subjective and, by itself, is insufficient. Computed tomography (CT) and MRI, while useful in demonstrating progressive brain inflammation and destruction, often fail to show any anatomical change in patients whose disease remits, particularly if surgery has been performed. $^{5}$

\section{Case report}

A 39-year-old, previously well, left-handed man presented in 1989 with generalised tonicclonic epileptic seizures. These were followed by intermittent left-sided facial simple partial motor seizures, complex partial seizures and a progressive left hemiparesis. His response to anti-epileptic medication was poor. In 1990 , brain MRI revealed increased signal around the right central sulcus on T2-weighted images. He had right frontal corticectomy and biopsy in 1990. Histology of the specimen showed persistent localised inflammation, perivascular lymphocytic infiltrates, astrogliosis, microglial nodules in grey and white matter, immune complexes in the affected brain tissue and neuronal loss with gliosis. These are changes typical of RE.

Although there was an initial mild improvement after surgery, his epilepsy and hemiparesis again worsened. Over the next few years he also developed progressive dysphasia, cognitive decline and a left homonymous hemianopia. $\mathrm{He}$ had right frontoparietal transection in 1995.

By June 1996 his seizures and hemiparesis were incapacitating. On MRI, there was increased T2 signal from most of the right frontal and parietal lobes associated with atrophy. Electroencephalography showed frequent right frontoparietal sharp wave discharges. Cerebrospinal fluid (CSF) contained 5 lymphocytes $/ \mathrm{mm}^{3}$. On semiquantitative interictal brain perfusion single photon emission computed tomography (SPECT) scan using ${ }^{99} \mathrm{Tc}^{\mathrm{m}}$ HMPAO (hexamethylene propylene amine oxime), there was markedly reduced perfusion of most of the right cerebral hemisphere (figure). In addition there were several contralateral focal perfusion defects.

He started treatment with prednisolone and hIVIg $(0.4 \mathrm{~g} / \mathrm{kg} /$ day for 5 days $)$. In total, he had six high-dose cycles of hIVIg at intervals of 4-6 
Figure Improvement in perfusion to the right frontal, temporal, and parietal lobes between baseline (left) and post-therapy scans (right)

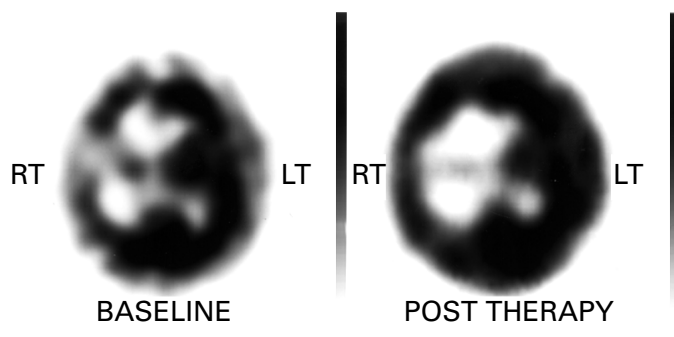

weeks. He then switched to monthly maintenance hIVIg infusions $(0.4 \mathrm{~g} / \mathrm{kg} /$ day $) .^{5}$

Around 4 months into treatment, he began to improve. His tonic-clonic seizures stopped and simple partial seizures reduced in severity and frequency. His hemiparesis recovered progressively, and he became independently mobile.

A second interictal brain perfusion SPECT scan was performed 8 months into treatment. We used spatial registration of both sets of SPECT images (MultiModality software, $\mathrm{Nu}-$ clear Diagnostics, UK) to compare the scans. Visually, the registered images show improvement in perfusion to right frontal, temporal and parietal lobes (figure).

We drew regions of interest over the four lobes of the cerebral hemispheres: frontal, temporal, parietal and occipital lobes. Uptake in each of these regions was compared with ipsilateral cerebellar uptake, which served as an internal reference. Occipital ratios are used when either or both cerebellar lobes have reduced perfusion. The lobar to cerebellar uptake ratios were compared with similar ratios calculated from normal data (summed multiple slices) obtained from SPECT scans of 22 healthy volunteers. Variation from this normal database is denoted by the number of standard deviations (SD). Since $95 \%$ of values lie within $2 \mathrm{SD}$ either side of the mean for the normal distribution, SD values of 2 or less are associated with a $\mathrm{p}$-value of $<0.05$.

In our patient, pre-treatment semiquantitative SPECT regional cerebral blood flow $(\mathrm{rCBF})$ data revealed a significant $(>2 \mathrm{SD}, \mathrm{p}<$ $0.05)$ reduction in perfusion to right frontal, temporal and parietal lobes and the left frontal lobe. Occipital ratios were used because the patient's right cerebellum showed reduced perfusion compared to the left cerebellum. Comparison of post-therapy values after standardisation revealed significantly improved perfusion to the right frontal (SD 6.65, p< 0.005 ) and right temporal (SD 3.53, p< 0.005 ), and to a lesser extent to the right parietal lobe (SD 1.73, p>0.05) (table). Eight months into treatment, CSF was normal. Serial brain MRI showed no change.

\section{Discussion}

In our patient, pre-treatment interictal brain SPECT scan showed perfusion defects predominantly in the clinically affected and surgically resected areas of the right cerebral hemisphere. The visual interpretation of hy-
Table Semiquantitative SPECT data: changes in regional perfusion after immunomodulatory treatment (figures in columns 2 and 3 represent standard deviations from normal database)

\begin{tabular}{lrcc}
\hline Occipital ratios & Baseline & Post-therapy & $\begin{array}{l}\text { Improvement } \\
\text { in perfusion }\end{array}$ \\
\hline Left cerebellum & 0.42 & 0.14 & -0.28 \\
Left temporal & -0.49 & 0.95 & 1.44 \\
Left parietal & 0.07 & 0.81 & -0.74 \\
Left frontal & -2.57 & -1.41 & 1.16 \\
Right cerebellum & -0.27 & 0.37 & 0.64 \\
Right temporal & -12.29 & -8.76 & 3.53 \\
Right parietal & -10.68 & -8.95 & 1.73 \\
Right frontal & -8.10 & -1.45 & 6.65 \\
\hline
\end{tabular}

poperfusion was confirmed by semiquantitative analysis of the rCBF data. Several methods have been developed to increase the sensitivity and reduce the variability of subjective visual reporting of SPECT. ${ }^{6}$ These techniques increase the reproducibility of reporting by comparing patient $\mathrm{rCBF}$ data with those from healthy control subjects. Moreover, serial independent data sets from a patient, here pre- and post-treatment, can be compared indirectly by comparing each data set independently with the same control data.

Perfusion brain SPECT is known to be a useful method to show functional changes associated with RE and many other types of encephalitis and encephalopathy. ${ }^{7-10}$ The usual findings are focal or multifocal perfusion defects. Typically, in RE, there is decreased perfusion covering confluent and extensive areas of the frontal, temporal and parietal lobes of one cerebral hemisphere. ${ }^{10}$ While this pattern was seen in our patient, an area of hypoperfusion was also noted in the clinically and MRI normal contralateral cerebral hemisphere. We do not have an explanation for this contralateral perfusion defect. Previous reports of bilateral perfusion SPECT defects in RE patients were ascribed to crossed diasthesis, ${ }^{10}$ although patients with RE affecting both cerebral hemispheres have been described. ${ }^{4}$ In other more widespread forms of encephalitis, however, multiple focal perfusion defects are commonly seen bilaterally. ${ }^{7}$ Thus, the pattern of decrease in $\mathrm{rCBF}$ is largely determined by the underlying pathology. By analogy, perhaps the contralateral changes in our patient represented subclinical disease.

This is the first demonstration of improvement in $\mathrm{rCBF}$ to the clinically affected brain area in RE in response to therapy. Reperfusion also occurred in regions of previous surgery. Moreover, increases in blood flow were accompanied by the suppression of seizures and striking recovery of functions associated with the right frontal, temporal and parietal lobes. These findings suggest that the potential for recovery persists even in patients with advanced RE and established brain destruction. Brain perfusion defects on SPECT can be produced by several pathogenic mechanisms including direct cellular damage caused by viruses, arteritis causing vessel occlusion, ${ }^{8}$ and toxic effects of enzymes and cytokines 
released from damaged brain cells and inflammatory cells. ${ }^{9} \mathrm{RE}$ is an antibody-mediated autoimmune disease characterised by inflammatory infiltrates containing activated lymphocytes, macrophages and glial cells. In our patient, improvement in regional cerebral perfusion was accompanied by resolution of CSF lymphocytosis suggesting that increased perfusion resulted from successful immunomodulation. We conclude that serial interictal perfusion brain SPECT scanning and semiquantification is a useful and objective method to monitor and validate the response to immunomodulatory treatment in patients with RE. It is a more sensitive method than structural brain imaging, which often fails to demonstrate any improvement in established destructive disease and is particularly difficult to interpret in a patient who has had extensive surgery.

1 Rasmussen T, Olszeweski J, Lloyd-Smith D. Focal seizures due to chronic localised encephalitis. Neurology 1958;8:435 45.

2 Villemure J-G, Andermann F, Rasmussen TB. Hemispherectomy for the treatment of epilepsy due to chronic encephalitis. In: Andermann F, ed, Chronic encephalitis and epilepsy: Rasmussen's syndrome. Boston: ButterworthHeinemann, 1991; pp 235-41.

3 Honavar M, Janota I, Polkey CE. Rasmussen's encephalitis and surgery for epilepsy. Dev Med Child Neurol 1992;34:314.

4 Hart YM, Cortez M, Andermann F, et al. The medical treatment of Rasmussen's syndrome: effect of high dose steroids and/or immunoglobulins in 19 patients. Neurology 1994;44:1030-6.

5 Leach JP, Chadwick D, Miles JB, Hart IK. Improvement in adult-onset Rasmussen's encephalitis with long-term immuadult-onset Rasmussen's encephalitis with long-term

6 Matsuda H, Tsuji S, Schuke $\mathrm{N}$, et al. A quantitative approach to technetium-99m hexamethylpropylene amine oxime. Eur f Nucl Med 1992;19:195-205.

\section{Summary points}

- although Rasmussen's encephalitis is common in children under 10 years, adults can also develop this disorder

- immunomodulatory therapies may be used to modify or halt progression of the disease

- serial MRI scans may show permanent brain damage

- serial perfusion brain SPECT scans can demonstrate improvement in established destructive disease and can be used to monitor and validate the response to immunomodulatory treatment

The patient's clinical response to hIVIg has been described previously.

7 Pohl P, Vogl G, Fill H, et al. Single photon emission tomography in AIDS demenia complex. F Nucl Med 1988;29: 1382-5

8 Tran Dinh Y, Mamo H, Cervoni J, et al. Disturbances in the cerebral perfusion of human immune deficiency virus-1 seropositive asymptomatic subjects: a quantitative tomographic study of 18 cases. 7 Nucl Med 1990;31:1601-4.

9 Gray F, Hurtrel M, Hurtrel B. Early central nervous system changes in human immunodeficiency virus infection. Neuropathol Appl Neurobiol 1993;19:3-5.

10 Hwang PA, Gilday DL, Spire J-P, et al. Chronic focal encephalitis of Rasmussen: functional neuroimaging studies with positron emission tomography and single photon emission tomography scanning. In: Andermann F, ed. Chronic encephalitis and epilepsy - Rasmussen's syndrome. Boston: Butterworth-Heinemann, 1991; pp 61-72. 


\title{
Eosinophilic fasciitis with pulmonary and pleural involvement
}

\author{
J W W Killen, G L Swift, R J White
}

\begin{abstract}
Summary
We report a case of eosinophilic fasciitis, with the unusual features of pulmonary and pleural involvement. Similar cases which involve the lungs have been reported after exposure to L-tryptophan, but there is no relevant drug history in this case.
\end{abstract}

Keywords: eosinophilic fasciitis; lung disease

A 50-year-old woman presented with increasing breathlessness of one month's duration without cough or wheeze. She described the sensation as different from her mild asthma which was well controlled on inhaled beclomethasone dipropionate and occasional salbutamol. There was no other drug history. In the past 2 weeks she had noticed the appearance of several nodules, first on her left leg and then a more diffuse swelling in her forearms and hands. Other relevant history was of night sweats for one week, and pain in the right calf.

On examination there were signs of small bilateral pleural effusions, and some inspiratory crackles at the left base. There was diffuse non-tender induration of both forearms and non-tender areas of induration on her legs, of about $5 \times 3 \mathrm{~cm}$. There was tendon swelling in her palms with triggering of fingers.

Initial investigations showed a normocytic anaemia, blood eosinophilia, raised inflammatory markers (C-reactive protein $95 \mathrm{mg} / \mathrm{l}$ (normal range $<10 \mathrm{mg} / \mathrm{l}$ ), plasma viscosity $1.92 \mathrm{cp}(1.5-1.72))$, and a restrictive pattern of ventilatory function. These results and the response to steroids are summarised in the table.

Submitted 16 April 1998 Accepted 5 July 1999
Chest X-ray confirmed small bilateral pleural effusions and in addition there was patchy parenchymal shadowing in both lower lobes. Pleural fluid was aspirated, which contained a large number of inflammatory cells, the majority of which were eosinophils. Eosinophils were markedly increased in bone marrow aspirate and trephine samples, and bronchial biopsy showed normal mucosa infiltrated with sheets of eosinophils.

Over the next 2 weeks her health deteriorated, the skin nodules and pleural effusions enlarged, and she developed a low grade pyrexia and a right foot drop. High resolution computed tomography (CT) of the chest showed pronounced interlobular and peribronchovascular nodular interstitial thickening, and bilateral pleural effusions, but no distortion of the lung architecture. Echocardiography was normal. Nerve conduction studies were normal, apart from suggesting a possible proximal S1 root lesion. Histology of deep skin and fascial biopsy from a lesion on the right forearm showed subcutaneous infiltration with eosinophils, predominantly perivascular in distribution, with focal fibrosis. The appearances were those of eosinophilic fasciitis.

She was started on enteric-coated prednisolone $30 \mathrm{mg}$ daily and she improved sufficiently for discharge from hospital within a week (see table). Six months later she is well, with no induration, no chest symptoms, and a normal chest X-ray. She is currently taking prednisolone $5 \mathrm{mg}$ daily and inhaled beclomethasone dipropionate $100 \mu \mathrm{g}$ bid.

\section{Discussion}

Eosinophilic fasciitis was first described by Shulman in $1974,{ }^{1}$ and shares some clinical features with progressive systemic sclerosis, into which it may progress. Its typical presentation is with tender swellings over the limbs, associated with arthralgia, hypergammaglobulinaemia and eosinophilia, but relative sparing of the viscera. The aetiology is unknown, but $50 \%$ of cases occur after strenuous exercise. The sex incidence is equal, and the mean age at onset is 47 years. Most cases resolve spontaneously within 3-5 years, and response to high-dose prednisolone is seen in about $70 \%$ of cases. $^{23}$

Restrictive pulmonary defect has been shown before but not to this degree, ${ }^{4-6}$ and there is no report of abnormal chest X-ray or diffusion capacity. Pleural effusions have not

Key : $\mathrm{Hb}=$ haemoglobin (normal range 11.5-16.5), Eos = eosinophil count $(<0.5), \mathrm{Plt}=$ platelet count $(150-400)$, Visc = plasma viscosity $(1.5-1.72)$, FEV1 $=$ forced expiratory volume in one second, FVC = forced vital capacity, DLCO = transfer factor for carbon monoxide 
previously been described. Silver et al described clinical and histological features consistent with but not identical to eosinophilic fasciitis as part of eosinophilia-myalgia syndrome after L-tryptophan ingestion. ${ }^{7}$ This disease was recognised in 1989 after an epidemic associated with L-tryptophan use as a diet supplement and antidepressant. The Centres for Disease Control case definition from the epidemic of 1989 comprises (1) peripheral blood eosinophil count greater than or equal to $1 \times 10^{9}$ cells $/ 1$, (2) generalised myalgia at some point during the illness severe enough to affect patients' ability to pursue daily activities, and (3) the absence of any infection or neoplasm to account for (1) or (2). A surveillance programme based on this definition found that, of 1075 cases identified, 3\% had no recollection of prior L-tryptophan ingestion, and a further $1 \%$ had not used L-tryptophan for over one month. ${ }^{8}$ During their illness, $59 \%$ of all cases had cough or dyspnoea. Of 718 patients who had a chest X-ray, $17 \%$ showed pulmonary infiltrates, there were pleural effusions in $12 \%$, and both in $8 \%$. Hibbs et al examined a register of eosinophilic fasciitis patients diagnosed prior to 1989 , and found that nine of 45 patients had taken L-tryptophan prior to disease onset, and six of these had dyspnoea. ${ }^{9}$ None of the 33 who had not taken L-tryptophan had

1 Shulman LE. Diffuse fasciitis with hypergammaglobulinaemia and eosinophilia in a new syndrome. $\mathcal{f}$ Rheumatol 1975;1(suppl):46

2 Maddison PJ. Eosinophilic fasciitis. Br F Rheumatol 1990;29: $81-2$.

3 Lakhanpal S, Ginsburg WW, Michet CJ, Doyle JA, Moore SB. Eosinophilic fasciitis: clinical spectrum and therapeutic response in 52 cases. Semin Arthritis Rheum 1988;17:221respon.

4 Caspi D, Fishal R, Varon M, Yona E, Baratz M, Yaron M. Multisystem presentation of eosinophilic fasciitis. Rheumato Rehab 1982;21:218-21.

5 Caperton EM, Hathaway DE, Delmer LP. Morphoea, asciitis and scleroderma with eosinophilia : a broad spectrum of disease. Arthritis Rheum 1976;19:792-3

6 Kent LT, Cramer SF, Moskowitz RW. Eosinophilic fasciitis clinical, laboratory, and microscopic considerations. Arthritis Rheum 1981;24:677-83. dyspnoea. The authors suggest that the presence or absence of pulmonary disease is a discriminant feature between eosinophilic fasciitis and eosinophilia-myalgia syndrome. However, our patient has no history of any exposure to drugs or preparations possibly containing L-tryptophan, and severe generalised myalgia was never a feature of her presentation.

The possible differential diagnoses of Churg-Strauss syndrome or hypereosinophilic syndrome were considered, in view of the combination of pulmonary eosinophilia and neurological disease, but the diffuse induration of the skin and the tendon involvement in our patient were typical of eosinophilic fasciitis. The proximal S1 root lesion is coincident, but it is unclear whether or not it is related to the eosinophilic fasciitis. Multifocal peripheral polyneuropathy has recently been described with eosinophilic fasciitis. ${ }^{10}$

In summary therefore, this patient had classical eosinophilic fasciitis with the unique features of eosinophilic infiltration of the lung and pleura. It is possible that this case represents a degree of overlap between syndromes, with distinctive features of its own, analogous to mixed connective tissue disease.

7 Silver RM, Heyes MP, Maize JC, Quearry B, VionnetFausset M, Sternberg EM. Scleroderma, fasciitis and eosinophilia associated with the ingestion of tryptophan. $N$ Engl f Med 1990;322:874-81.

8 Swygert LA, Maes EF, Sewell LE, Miller L, Falk H, Kilbourne EM. Eosinophilia-myalgia syndrome. Results of national surveillance. FAMA 1990;264:1698-703.

9 Hibbs JR, Mittleman B, Hill P, Medsger TA. L-Tryptophanassociated eosinophilic fasciitis prior to the 1989 eosinophilia-myalgia outbreak. Arthritis Rheum 1992;35: 299-303.

10 Satsungi J, Donaghy M. Multifocal peripheral neuropathy in eosinophilic fasciitis. F Neurol 1992;239:91-2. 
Department of

Medicine, Armed

Forces Medical

College, Pune 411040,

India

A S Kashyap

Cardiothoracic

Centre, Goliban

Maidan, Pune 411040,

India

S Kashyap

Submitted 1 June 1999 Accepted 14 June 1999

\title{
Increased prevalence of impaired hearing in patients with type 2 diabetes in western India
}

\author{
A S Kashyap, S Kashyap
}

\author{
Summary \\ In an out-patient study of 1344 general \\ medical patients over 50 years old in \\ India, the prevalence of hearing aid use \\ was found to be significantly greater in \\ type 2 diabetics.
}

Keywords: diabetes; deafness; India

There has been persistent concern about the increased incidence of hearing loss in diabetes. The literature is scanty on this association, ${ }^{12}$ and there are no studies from the Indian subcontinent. We conducted an out-patient study on the prevalence of deafness, defined as the use of a hearing aid, in 1344 general medical patients over 50 years of age, with and without diabetes.

The total number of diabetic patients was 120 , their mean age ( \pm SD) was $64 \pm 6$ years. The number of patients using a hearing aid are shown in the table. The prevalence of hearing aid use in the entire group was the same as in the local population as a whole. Within each age group, the prevalence was increased in dia-

\footnotetext{
1 Dalton DS, Cruickshanks KJ, Klein R, Klein BEK, Wiley TL. Association of NIDDM and hearing loss. Diabetes Care 1998;21:1540-4.
}

Table Number of patients using a hearing aid in a group of 1344 general medical patients in India

\begin{tabular}{lll}
\hline Age (years) & All patients (\%) & Diabetic patients (\%) \\
\hline $50-59$ & $18(3)$ & $6(17.2)$ \\
$60-69$ & $32(7.2)$ & $9(23.2)$ \\
$>70$ & $45(17.1)$ & $14(34)$ \\
Total & $95(7.0)$ & $29(24)$ \\
\hline
\end{tabular}

betic compared with non-diabetic patients $(\mathrm{p}<0.001)$. Deaf diabetic patients were older $(71 \pm 8.6$ vs $65.2 \pm 7.3$ years, $\mathrm{p}<0.01$ adjusted for sex), and they had a longer duration of diabetes compared to those who did not use a hearing aid $(10.2 \pm 6.2$ vs $8.4 \pm 6.1$ years, $\mathrm{p}=0.10$ adjusted for sex and age). HbA $1 \mathrm{c}$ levels during the last year were slightly higher in the deaf patients $(7.2 \pm 1.2$ vs $6.9 \pm 1.1 \%$, $\mathrm{p}>0.1$ adjusted for age and sex). There was no difference in prevalence of neuropathy, retinopathy or nephropathy.

We agree with findings of Dalton et $a l^{1}$ and Stolk et $a l,{ }^{2}$ and hypothesise that deafness might represent a diabetic complication in type 2 diabetic patients.

2 Stolk RP, Boomsma LJ. Prevalence of deafness is increased in patients with type 2 diabetes in the Netherlands (letter). Diabetes Care 1999;22:180. 


\title{
Prolonged hypoglycaemia following surgical removal of phaeochromocytoma
}

\author{
E B Jude, C B R Sridhar
}

\begin{abstract}
Summary
We report a 34-year-old woman with secondary hypertension diagnosed with a left-sided phaeochromocytoma. Excision of the tumour resulted in recurrent hypoglycaemia up to 6 days post-operatively. We discuss the pathogenesis of hypoglycaemia following removal of the tumour and put forward hypothesis for the recurrent hypoglycaemia.
\end{abstract}

Keywords: phaeochromocytoma; hypoglycaemia

Phaeochromocytoma is a not uncommon cause of secondary hypertension and treatment consists of surgical excision. Transient hypoglycaemia has been reported previously after removal of the tumour. We report a patient with recurrent hypoglycaemia up to 6 days post-operatively, who required monitoring and glucose infusion for a prolonged period. So far, no reports of recurrent hypoglycaemia have been published in the literature. In this case report we highlight the need for prolonged monitoring of patients following surgical excision of a phaeochromocytoma.

\section{Case report}

A 34-year-old-woman presented with headache, giddiness, palpitations, excessive sweating and chest pain for 6 months. Hypertension was diagnosed just prior to admission by her general practitioner but she was not commenced on treatment. Examination revealed an anxious tense woman, supine blood pressure (BP) 160/120 $\mathrm{mmHg}$ and lower limb BP 170/130 $\mathrm{mmHg}$. There were no café-au-lait spots, neurofibromas or neurocutaneous lesions. Abdominal, respiratory and cardiovascular system examination and fundi were normal. There was no bruit over the renal arteries. She did not have a goitre. With a clinical diagnosis of phaeochromocytoma, the patient was investigated. Full blood count, renal function, serum calcium, electrolytes, fasting blood glucose, thyroid function tests, electrocardiogram and chest X-ray were normal. Urinary catecholamines was 200 $\mu \mathrm{g} / 24 \mathrm{~h}$ (normal $100-150 \mu \mathrm{g} / 24 \mathrm{~h}$ ) and vanillyl mandelic acid was $24.15 \mathrm{mg} / 24 \mathrm{~h}(2.5-5 \mathrm{mg} / 24$ h). Abdominal ultrasound revealed a left-sided suprarenal lesion measuring $6 \times 6 \times 5 \mathrm{~cm}$ with no masses along the sympathetic chain. The patient was commenced on phenoxybenzamine $10 \mathrm{mg}$ 6-hourly and propranolol $40 \mathrm{mg}$ bid. Pre-operatively her BP was 130/90 mmHg.

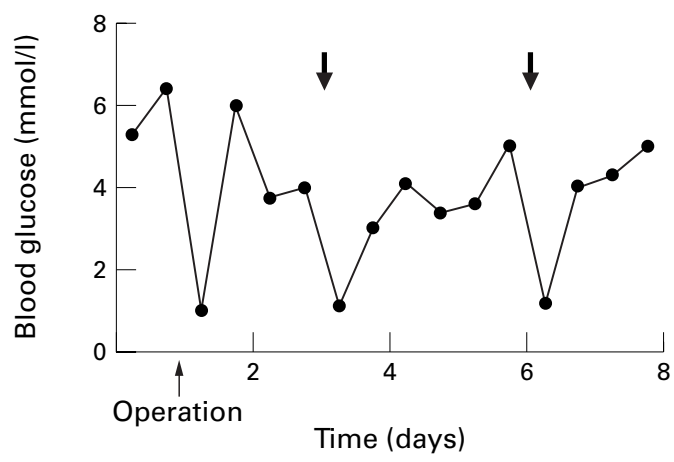

Figure Pre- and post-operative blood glucose levels showing recurrent hypoglycaemia after removal of phaeochromocytoma (solid arrow indicates where glucose infusion was stopped)

The patient underwent surgery under cover of intravenous sodium nitroprusside and propranolol. A left-sided suprarenal tumour was removed and histology confirmed a phaeochromocytoma. There were no complications during surgery and blood loss was average. Intra-operatively she received 1.51 of $5 \%$ dextrose and one unit of blood.

Two hours post-operatively, the patient lapsed into a stuporous state and on investigation her blood glucose was $1.1 \mathrm{mmol} / 1$. She was given an immediate intravenous infusion of 50

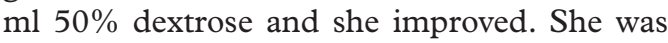
started on $10 \%$ dextrose infusion with $50 \mathrm{ml}$ $50 \%$ dextrose bolus every 4 hours. On the third day, oral feeds were introduced and intravenous fluids were discontinued. Three hours later she became unresponsive and her blood glucose was $1.2 \mathrm{mmol} / \mathrm{l}$. She was recommenced on a continuous infusion of $10 \%$ dextrose after a bolus of $50 \%$ dextrose. On the sixth day she had a third episode of hypoglycaemia (blood glucose $1.3 \mathrm{mmol} / \mathrm{l}$ ) when the dextrose infusion was stopped, which was recommenced and continued for a further $48 \mathrm{~h}$ and then discontinued (figure). She did not have further episodes of hypoglycaemia in hospital. Insulin assays were in the normal range but at the time of blood sampling she was normoglycaemic. She was followed up regularly over the next 18 months and had not suffered further hypoglycaemic episodes. A prolonged fast and abdominal ultrasound done at follow-up were normal.

\section{Discussion}

This is the first case of recurrent hypoglycaemia following surgical removal of a phaeochro- 
mocytoma. Transient hypoglycaemia (within 12 hours) has been reported previously in the literature. ${ }^{12}$ Various metabolic factors give rise to hypoglycaemia after this surgery. Adrenaline infusion has been shown to inhibit insulin secretion and stimulate glucagon secretion in normal subjects. ${ }^{34}$ Sudden withdrawal causes reversal of these effects and a rebound hyperinsulinaemia inappropriate to the prevailing blood glucose concentration. Previously, it has been suggested that endogenous insulin secretion is suppressed by the increased circulating catecholamines in patients with phaeochromocytoma, and that excessive rebound secretion of insulin occurs after the removal of the tumour. ${ }^{2}$ In addition, prolonged exposure to adrenaline may delay the recovery of the hypoglycaemic response to glucagon. Hypercatecholaemia depletes liver glycogen and inhibits glucose uptake, thus impairing the body's response to hypoglycaemia. ${ }^{5}$ These factors may explain the occurrence of transient hypoglycaemia after removal of phaeochromocytoma.

Insulinoma is a possible cause for recurrent hypoglycaemia but insulin assays were normal in our patient and after the sixth day she did not have further episodes of hypoglycaemia. Furthermore, she did not develop hypoglycaemia during prolonged fast and in the 18 months follow-up after surgery she was healthy and doing well, which probably excludes insulinoma as a possible cause for her recurrent hypoglycaemia in the immediate post-operative period. Other possible explanations for the hypoglycaemia are:

- the dextrose infusions given to the patient in the post-operative period were insufficient to replenish the depleted glucose and glycogen

1 Chambers S, Espiner EA, Donald RA, Nichols MG. Hypoglycaemia following removal of phaeochromocytoma: case report and review of the literature. Postgrad Med $f$ 1982;58:503-6.

2 Akiba M, Kodama T, Ito Y, Obara T, Fujimoto Y. Hypoglycaemia induced by excessive rebound secretion of insulin after removal of phaeochromocytoma. World f Surg 1990;14:317-24

3 Porte D Jr, Graber AL, Kuzuya T, Williams RH. The effect of epinephrine on immunoreactive insulin levels in man. $\mathcal{F}$ Clin Invest 1966;45:228-36.

\section{Learning points}

- phaeochromocytoma is an uncommon cause of secondary hypertension

- transient hypoglycaemia is known to occur after surgical excision of a phaeochromocytoma

- recurrent hypoglycaemia after surgery may also occur

- all patients undergoing surgical excision of a phaeochromocytoma must be monitored for prolonged period (eg, 7 days) to prevent the complications of hypoglycaemia

stores in the liver resulting from a prolonged hyper-catecholaemic state

- catecholamine levels in the body may remain elevated for up to a week post-operatively ${ }^{6}$

- studies have shown a transient hyperinsulinaemic phase, ${ }^{12}$ but a prolonged hyper-insulinaemic phase may also exist.

Patients undergoing major surgery are monitored in high-dependency or intensive-care units and are usually on narcotic analgesics, as a result of which they may be drowsy. They could also be drowsy as a result of the anaesthesia itself. In such patients hypoglycaemia may be missed unless it is specifically looked for. Therefore, there must be increased awareness of the possibility of hypoglycaemia in patients undergoing removal of a phaeochromocytoma. In addition, these patients may have further hypoglycaemia up to a week postoperatively. The cause of recurrent hypoglycaemia needs further investigation, but meanwhile such patients must be monitored for up to a week to avoid the complications of hypoglycaemia.

4 Gerich JE, Karam J, Forsham P. Stimulation of glucagon secretion by epinephrine in man. F Clin Endocrinol Metabol 1973;37:479-81

5 Ellis, S. Relation of biochemical effects of epinephrine to its vascular effects. Pharmacol Rev 1959;59:469-79.

6 Daly PA, Landsberg L. Phaeochromocytoma: diagnosis and management. Bailliere's Clin Endocrinol Metabol 1992;6:43166. 